\title{
Optimization of the formulation of a preservation medium for grafts in view of their subsequent transplantation
}

\author{
Neyla Ben Gdara* and Lotfi Bitri \\ Department of Biology, University of Tunis El Manar, Faculty of Sciences of Tunis, University campus 2092, El Manar, Tunis, Tunisia
}

\begin{abstract}
Liver transplantation is associated with hepatocellular damage to the transplant outcome known as ischemia/reperfusion (I/R) syndrome. Despite advances in liver storage, complications associated with cold injury still limit the optimal use of transplantation as a therapeutic intervention in the event of graft failure. One of the therapeutic challenges is to develop new treatments to protect and better preserve liver tissue damaged by a sequence of ischemia/reperfusion.
\end{abstract}

\section{Introduction}

Liver failure is a serious disease that could lead to complications inducing morbidity and mortality. Liver failure is defined as a lack of oxygen $\left(\mathrm{O}_{2}\right)$ and nutrients to the liver cells, causing progressive destruction of liver tissue. It is the result of partial or total obstruction of part of the hepatic arterial network, which is then responsible for the ischemia of the liver tissue. Therefore, the only curative treatment for most serious liver diseases is liver transplantation [1-3].

Organ transplantation was described in the early 20th century in the development of the first successful vascular anastomosis technique by Carell [4]. In 1954, the human kidney was successfully transplanted by Murray [5]. In 1963, Thomas Starlz performed the first successful liver transplant [6]. However, the survival rate was initially not very high; it was considerably increased in the 1980s, in particular by the improvement of techniques for the removal and preservation of implants, and especially by the introduction of immunosuppressants with the discovery of cyclosporin [7]. Organ transplantation is an effective therapy for most pathologies leading to an irreversible loss of vital organs. Different metabolic changes take place during different phases of liver transplantation, characterized by diverse and complex cellular, molecular and biochemical alterations known as the ischemia/ reperfusion syndrome $(\mathrm{I} / \mathrm{R})[8,9]$. During the transplantation process, the hepatic graft may be exposed to 3 types of ischemic episodes followed by reperfusion. Cold Ischemia follows the preservation of the liver in a hypothermic environment after removal. The aim of this practice is to reduce the metabolic activity of hepatic graft prior to implantation and reperfusion in the receiver. During cold ischemia, the hepatic graft undergoes a serious damage associated with a reduction of cellular ATP levels, and an enhancement of cytosolic calcium levels [10]. Warm ischemia applies in cases of hemodynamically unstable donors; it is practiced in normothermal conditions. Parenchymal cells are more sensitive to this type of ischemia in which oxidative stress and mitochondrial dysfunction predominate [11]. The third case: relative warm ischemia "rewarming". It consists of exposing the graft to body temperature during the preparation of vascular anastomoses and its manipulation. Reperfusion is the re-circulation of blood to the receiver. Restoration of blood flow during reperfusion has deleterious consequences for ischemic tissue [12]. Previous studies have shown that ischemia/reperfusion lesions have a major impact on the occurrence of acute rejection, on functional recovery of graft, and on graft survival [13].

\section{Hypothermic Conservation}

The graft cold preservation is one of the main principles of organ persistence that prolongs the preservation time and improves the quality of the graft.

The graft's viability during its ischemic transfer from donor to receiver is based mainly on the hypothermia by rinsing the graft in situ with a preservation solution $\left(+4^{\circ} \mathrm{C}\right)$, then it will be immersed in the same solution $\left(+4^{\circ} \mathrm{C}\right)$ for a certain period of time. Hypothermic organ preservation is the imperative condition for prolonged graft preservation. Hypothermia appears to be effective by blocking partially hydrolytic enzyme activity (phospholipases, proteases or endonucleases). However, hypothermia alone is not sufficient to prolong the viability of the organ graft. Indeed, a second important element of organ preservation is the use of a preservation solution. The success of organ preservation is highly dependent on the composition of the preserving liquid used.

Overall, two important factors can be identified for optimal organ preservation; Hypothermia which decreases the rate of catabolism of metabolites and structural components of cells and The conservation solution: vector of hypothermia, allows the establishment of an appropriate chemical environment, due to the presence of metabolites and inhibitors that act on the reactions of degradation, responsible for irreversible damages of the graft and thus allow the restoration of normal metabolism.

${ }^{*}$ Correspondence to: Neyla Ben Gdara, Department of Biology, University of Tunis El Manar, Faculty of Sciences of Tunis, University campus 2092, El Manar, Tunis, Tunisia, Tel: 002162061009; E-mail: neylabengdara@yahoo.fr

Key words: ischemia/reperfusion, cold ischemia, preservation solutions, oxidaive stress, hypothermic conservation

Received: November 13, 2020; Accepted: November 22, 2020; Published: November 25, 2020 


\section{Preservation Solutions}

There are non-colloidal conservation solutions whose composition is devoid of oncotic support (macromolecules). The non-colloidal solutions that have been used the most are: Celsior, HTK Custodiol and Collins. The Collins solution was developed in 1970 by Collins and associates and was later modified into the EuroCollins solution [14]. This solution contains magnesium for membrane stability, phosphate for proton buffering, a high concentration of potassium, and glucose which acts as an oncotic carrier. The high concentrations of potassium and magnesium in Collins' solution result in the precipitation of magnesium phosphate. These crystals are toxic to the graft, which leads to the modification of the Collins solution to a magnesium-free EuroCollins solution. The EuroCollins solution is the first specifically developed solution for kidney conservation [6]. Although this solution is effective for kidney transplant preservation, it is not suitable for liver preservation because of its high glucose concentration. Indeed, due to the permeability of hepatocytes to glucose, it penetrates into the cells where its phosphorylation into glucose-6-phosphate is activated by a glucokinase and will be rapidly metabolized into lactic acid. Under these conditions, glycolysis is not subject to negative feedback, causing an accumulation of $\mathrm{H}^{+}$and lactate ions responsible for severe cellular acidosis. On the other hand, in the kidney, the phosphorylation of glucose is dependent on a hexokinase which is negatively back-checked by glucose-6-phosphate.In the kidney; on the other hand, the phosphorylation of glucose is dependent on a hexokinase which is negatively back-checked by glucose-6-phosphate. The self-regulation of glucose metabolism explains the better efficacy of the EuroCollins solution for renal graft [14]. The Celsior solution and HTK (Histidine Tryptophan Ketoglutarate) are the most widely used in the preservation of liver transplants. Both solutions have a low viscosity and low potassium concentration. Histidine and tryptophan in the HTK solution act as a buffer, ketoglutarate acts as a metabolite. The Celsior solution contains lactobionic acid. The latter plays the role of osmotic agent and iron and $\mathrm{Ca}^{++}$chelator, promoting the generation of oxygenated free radicals (Oxygenated Free Radicals) harmful to the preserved organ.

Colloidal solutions include the University of Wisconsin (UW) solution, the Institut Georges Lopez solution (IGL-1) and the Polysol solution. The UW solution (or Belzer solution) has transformed organ preservation [15]. This solution ensures: maintenance of osmotic pressure not by a metabolically active agent such as glucose, but, more efficiently, by the addition of metabolically inert substrates such as lactobionate and raffinose. Glucose is also omitted from the composition to avoid the production of lactic acid and hydrogen ions; the presence of a colloid, in this case hydroxyl ethyl starch (HES), which is a stable and non-toxic agent, prevents the expansion of extracellular space. Its adjunction appears fundamental in case of prolonged conservation; the addition of antioxidants (glutathione, allopurinol) and precursors of ATP synthesis at the time of reperfusion (adenosine, phosphate). This solution allows storage times of 12-15 hours for the pancreas and liver, and up to 24-36 hours for the kidney $[15,16]$. However, the absence of a specific component to limit the deleterious effect of the intracellular calcium influx into the UW solution as well as its low sodium concentration, inherent to its intracellular formulation, promotes the accumulation of $\mathrm{Ca}^{++}$ions. Besides its impermeable properties, lactobionate would help to chelate calcium. This action would limit the activity of calcium-dependent enzymes such as proteases and phospholipases, which are involved in cell disintegration. Lactobionate could also chelate iron and thus limit oxidative damage during reperfusion. This liquid remained, for a very long time, as the reference for the conservation and preservation of abdominal organs (17). The IGL-1 solution is inspired by the principles of the two solutions UW and Celsior [17]. It combines the extracellular character of Celsior, by inversion of the electrolyte ratio, and the presence of a colloid as in UW, polyethylene glycol (PEG, $35 \mathrm{KDa}$ ) replacing HEA and significantly reducing solution's viscosity. Several experimental studies have shown that PEG in the IGL-1 solution significantly improved the quality of kidney and liver conservation $[13,17]$. This beneficial effect of PEG is manifested by a decrease in lipid peroxidation lesions and apoptosis $[18,19]$. This colloid participates in improving the immune tolerance of the graft by limiting rejection through an immunomasking effect by spontaneously binding to surface cells, protecting the organ from interactions with the immunocompetent cells of the recipient. Experimental [20] and clinical studies [21,22] on liver and kidney transplantation have shown the beneficial effects of IGL-1 against apoptosis, stress of the endoplasmic reticulum and immune response. Indeed, it further promotes nitric oxide (NO) synthesis through the activation of endothelial nitric oxide synthase (eNOS), thus reducing $\mathrm{I} / \mathrm{R}$ lesions and protecting microcirculation [23]. Polysol is a lowviscosity solution, which however maintains a high oncotic pressure due to the incorporation of PEG. In addition, Polysol is highly enriched with antioxidants (free radical scavengers), precursors of ATP synthesis, amino acids, vitamins and nutrients, ingredients whose common role is to counteract the adverse effects of hypothermia during the storage phase in order to limit reperfusion damage. In experimental situations $[24,25]$ as well as on the clinical level [26] promising results have been reported for the liver and kidney after storage in cold ischaemia or by hypothermic perfusion. One of the challenges of therapeutics is to develop new treatments to protect and better preserve liver tissue damaged by a sequence of ischemia/reperfusion.

\section{Modification of preservation solutions}

The addition of certain molecules to the preservation solution has been the subject of various experimental studies.

Anti ischaemic drug: Previous studies on renal [27,28], hepatic $[29,30]$ and cardiac $[31,32]$ models have demonstrated the antioxidant action of trimetazidine (TMZ), an anti-ischaemic drug. The addition of TMZ to the UW solution was tested in the livers of steatotic and non-steatotic rats after hypothermic storage and ex vivo perfusion [23]. Enrichment of the UW solution with TMZ reduced liver damage by decreasing microcirculatory dysfunction, oxidative stress and mitochondrial damage. Under the same experimental conditions, supplementation of IGL-1 solution with TMZ offered better liver graft preservation than IGL-1 solution alone and induced significant activation of the hypoxia-inducible factor- $1 \alpha$ (HIF $1 \alpha$ ) and increased NO production [33]. The benefits of TMZ have been clinically demonstrated in patients undergoing vascular clamped liver surgery [34]. This suggests that TMZ could be used as an additive in commercial preservation.

Hormone: Melatonin (ML), a hormone produced by the pineal gland in a circadian manner, has been shown to be very beneficial in protecting and better preserving both steatotic and non-steatotic rat livers from I/R damage when added to IGL-1 preservative solution. Indeed, ML ensures the protection of the liver graft by overexpressing endothelial NO synthase (e-NOS) and Heme Oxygenase-1. These protective effects of $M L$ on liver graft preservation were further enhanced by the addition of TMZ to the IGL-1 + ML solution [30].

Proteosome inhibitors: The addition of epoxomycin, a proteosome inhibitor, to UW solution reduces cardiac edema and preserves the 
structural integrity of post-ischemic cardiomyocytes [35]. At liver graft level, it has been shown that the addition of Bortezomide (BRZ) and MG132 to UW solution improved the preservation of steatotic and non-steatotic liver grafts, and that BRZ would be more effective than MG132 [30]. The addition of BRZ to IGL-1 also showed protective effects that were partially mediated by AMPK activation and Akt/ mTOR signaling [36].

HEMO2 life: A French study investigated an extracellular haemoglobin called M101 (produced by HEMARINA, Morlaix), isolated from the marine worm larva Arenicola marina, and which was developed under the product name HEMO2 life as an additive to preservative solutions during hypothermic storage of graft [37]. M101 has a high affinity for oxygen and can carry up to 156 oxygen molecules compared to the four oxygen molecules carried by human hemoglobin [38]. Oxygen release occurs on a gradient without any allosteric effect, and according to the p50 of the molecule [39]. The p50 reflects the af function of haemoglobin in oxygen and the value corresponds to the oxygen tension at which $50 \%$ of the $\mathrm{O}_{2}$ binding sites are saturated. This is a crucial advantage of M101 over other types of hemoglobin-based oxygen carriers (HBOC). The p50 of M101 is $7 \mathrm{~mm} \mathrm{Hg}$. The oxygen storage capacity of the molecule is maintained until the $\mathrm{pO}_{2}$ exceeds $7 \mathrm{~mm} \mathrm{Hg}$, at which time a severe hypoxic environment causes the passive release of oxygen, providing to the cell a sufficient supply of oxygen. When tissues consume the oxygen present in the preservative solution, the $\mathrm{pO}_{2}$ decreases below the $\mathrm{p} 50$ of $\mathrm{M} 101$, releasing the oxygen and providing a binding site for an additional oxygen molecule. As extracellular hemoglobin, M101 has intrinsic Cu/Zn-superoxide dismutase (SOD) activity, which protects against potential damage caused by heme protein-associated ROS or ischemia-bound ROS released into solution. M101 is also active between 4 and $37^{\circ} \mathrm{C}$, which is an appropriate temperature for multiple types of organ preservation. M101 is derived from a marine poiki-lothermal invertebrate which is not able to control its body temperature and undergoes strong seasonal and external daily temperature fluctuations. The efficacy and safety of M101 in organ preservation has been shown to improve graft function in clinical studies of renal and facial transplantation and in preclinical studies of the kidney, lung and heart $[40,41]$. In addition, the molecule can simply be added to the preservation solution either under static storage or with mechanical perfusion $[42,43]$.

Nitric oxide: In previous work we have demonstrated a dosedependent effect of the presence of NO in the liver graft preservation solution. Indeed, at a concentration of $50 \mathrm{nM}$, NO had an optimal protective effect on the graft, whereas at a high concentration of 1000 $\mathrm{nM}$, it was harmful and induced oxidative stress $[44,45]$.

Recently, we demonstrated the effect of the addition of phycocyanin, a molecule extracted from cyanobacteria (Spirulina platensis), to liver graft preservation solution during an ischemia/reperfusion episode, in order to better preserve liver's structural architecture and improve its regenerative capacities after damages caused by the oxidative stress that hepatocytes undergo. During this study, a perfused isolated liver model (FIP) was developed to evaluate the influence of hypothermic preservation on the quality of liver transplants and to test the effect of the addition of phycocyanin in the same preservation solution on the functional recovery of the liver and the reduction or even inhibition of the sequels induced by the oxidative stress generated during the ischemia/reperfusion process. Hypothermic conservation induces lesions in rat liver which are marked by disturbances of metabolic and cellular parameters: indeed, the levels of hepatotoxicity parameters (Alanine aminotransferase (ALAT), Aspartate aminotransferase
(ASAT) and Alkaline Phosphatase (PAL)) in the perfusate are significantly increased, indicating cellular lesions. Metabolic parameters (glucose, cholesterol and triglycerides) were also increased in the perfusate. These increases are more pronounced if the conservation period is longer. I/R process is considered to be a major factor in the induction of synthesis and activity of oxidative stress parameters. In fact, hepatic levels of Malondialdehyde (MDA), Reduced Glutathione (GSH), Gluthation Peroxidase (GPx) and Gluthation S-transferase (GST) were significantly increased in parallel with a reduction in thiol levels. This confirms a stress state noted during I/R.

The addition of phycocyanin to the preservative solution corrects for induced I/R consequences. The evolution of the above parameters seems to be reversed with the presence of phycocyanin by reducing hepatotoxicity (decrease in transaminase and alkaline phosphatase levels), reduction of hepatocellular edema which are responsible for the release of glucose, cholesterol and triglycerides in the perfusate.

The introduction of phycocyanin to the preservative solution significantly reduces the synthesis of oxidative stress parameters (MDA, GSH, GPx and GST) and increases the level of thiols tissue. The expression of mitochondrial proteins normally induced by oxidative stress is stimulated during hypothermic conservation but is reduced with the addition of phycocyanin to the conservation solution, which confirms the protective effect of this pigment against cellular damage affecting in particular mitochondrial DNA by genetic mutations or deletions caused by oxidative stress induced by I/R.

The presence of phycocyanin at different doses in the preservative solution significantly improves the functional quality of the liver graft at the metabolic, enzymatic, cellular and molecular levels.

The graft is thus better preserved for transplantation and regeneration in the receiver, avoiding the sequelae of the damage observed at all stages of transplantation. The addition of phycocyanin in the preservation solution would be more effective in the preservation of liver transplants.

\section{Conclusion}

Taking into account these findings, it is clear that the choice and the improvement of the composition of the preservative solution are necessary to optimize the quality of the preserved graft and to limit ischemia/reperfusion injuries. Enrichment of preservative solutions with pharmacologically active substances may protect livers from cold I/R injuries.

\section{Acknowledgement}

None.

\section{References}

1. Parsons RF, Guarrera JV (2014) Preservation solutions for static cold storage of abdominal allografts: which is best? Curr Opin Organ Transplant 19:100-107. [Crossref]

2. Ravikumar R, Leuvenink H, Friend PJ (2015) Normothermic liver preservation: A new paradigm? . Transpl Int.; 28: 690-699. [Crossref]

3. Barbas AS, Goldaracena N, Dib MJ, Selzner M (2016) Ex-vivo liver perfusion for organ preservation: Recent advances in the field. Transplant Rev (Orlando) 30: 154160. [Crossref]

4. Carell A (1908) Results of the transplantation of blood vessels, organ and limbs JAMAS 51: 1662 .

5. Murray JE, Tilney NL, Wilson RE (1976) Renal transplantation: a tweety-five-year experience. Ann Surg 184: 565. [Crossref] 
6. Blankensteijn JD, Terpstra OT (1990) Liver preservation: The past and the future. $J$ Hepatol 13: 1235-1250. [Crossref]

7. Starlz TE, Klintmalm GB, Porter KA, Iwatsuki S, Schröter GP (1981) Liver transplantation with use cyclosporine and prednisone. N Engl J Med 305: 266-269. [Crossref]

8. Brass CA, Nunes F, Negpal R (1994) Increased oxyradical production during reoxygenation of perfused rat liver. Transplantation 58:1329-1335. [Crossref]

9. Jamieson NV (1991) Improved preservation of liver for transplantation. Aliment pharmacol ther 5: 91-104. [Crossref]

10. Auger S, Vallerand D, Hadded PS (2003) Cold preservation-warm reperfusion perturbs cytosolic calcium homeostasis in rat liver sinusoidal endothelium cells. Liver Transpl 9: 150-159. [Crossref]

11. Mochida S, Arai M, Ohno A, Masaki N, Ogata I, et al. (1994) Oxidative stress in hepatocytes and stimulatory state of Kupffer cells after reperfusion differ between warm and cold ischemia in rats. Liver 14: 234-240. [Crossref]

12. Eschwège $P$ (2005) What information is provided by transplantation on renal protection? Ann Fr Anesth Reanim 24: 182-193. [Crossref]

13. Badet L, Ben Abdennebi H, Petruzzo P (2005) Evaluation de IGL-1, une nouvelle solution de conservation d'organe: résultats pré-cliniques en transplantation rénale. Progrès en Urologie 15: 481-488.

14. Belzer FO, Southard JH (1988) Principles of solid-organ preservation by cold storage. Transpl 45: 673-676. [Crossref]

15. Yoglu M, Sollinger HW, Stratta RJ (1988) Extended preservation of the liver for clinical transplantation. Lancet 331: 617-619. [Crossref]

16. Ploeg RJ, Boudjema K, Marsh D (1992) The importance of a colloid in canine pancreas preservation. Transpl 53: 735-741. [Crossref]

17. Ben Abdennebi H, Steghens JP, Hadj-Aissa A (2002) A preservation solution with polyethylene glycol and calcium: a possible multiorgan liquid. Transpl Int 15: 348-345.

18. Faure JP, Hauet T, Han Z (2002) Polyethylene glycol reduces early and long-term cold ischemia-reperfusion and renal medulla injury. J Pharmacol Exp Ther 302: 861-870. [Crossref]

19. Mack JE, Kerr JA, Vreugdenhil PK, Belzer FO, Southard JH (1991) Effect of polyethylene glycol on lipid peroxidation in cold-stored rat hepatocytes. Cryobiology 28: 1-7. [Crossref]

20. Mosbah IB, Saidane D, Peralta C, Roselló-Catafau J, Abdennebi HB (2005) Efficacy of polyethylene glycols in University of Wisconsin preservation solutions: a study of isolated perfused rat liver. Transplant Proc 37: 3948-3950. [Crossref]

21. Codas R, Petruzzo P, Morelon E (2009) IGL-1 solution in kidney transplantation: first multi-center study. Clin Transplant 23: 337-342. [Crossref]

22. Dondéro F, Paugam-Burtz C, Danjou F, Stocco J, Durand F, et al. (2010) A randomized study comparing IGL-1 to the University of Wisconsin preservation solution in liver transplantation. Ann Transplant 15: 7-14. [Crossref]

23. Ben Mosbah I, Casillas-Ramírez A, Xaus C, Serafin A, Roselló-Catafau J, et al. (2006) Trimetazidine: is it a promising drug for use in steatotic grafts? World $J$ Gastroenterol 12: 908-914. [Crossref]

24. Bessem M, Doorschodt BM, van Vliet AK, van Gulik TM (2005) Improved rat liver preservation by hypothermic continuous machine perfusion using polysol, a new, enriched preservation solution. Liver Transpl 11: 539-546. [Crossref]

25. Yagi S, Doorschodt BM, Afify M (2011) Improved preservation and microcirculation with POLYSOL after partial liver transplantation in rats. J Surg Res 167: 375-383. [Crossref]

26. Schreinemachers MC, Bemelman FJ, Idu MM (2013) First clinical experience with polysol solution: pilot study in living kidney transplantation. Transplant Proc 45: 3845. [Crossref]
27. Singh D, Chopra K (2004) Effect of trimetazidine on renal ischemia/reperfusion injury in rats. Pharmacol Res 50: 623-629. [Crossref]

28. Mahfoudh-Boussaid A, Zaouali MA, Hauet T (2012) Attenuation of endoplasmic reticulum stress and mitochondrial injury in kidney with ischemic postconditioning application and trimetazidine treatment. J BiomedSci 19: 71. [Crossref]

29. Elimadi A, Settaf A, Morin D (1998) Trimetazidine counteracts the hepatic injury associated with ischemia-reperfusion by preserving mitochondrial function. $J$ Pharmacol Exp Ther 286: 23-28. [Crossref]

30. Zaouali MA, Boncompagni E, Reiter RJ (2013) AMPK involvement in endoplasmic reticulum stress and autophagy modulation after fatty liver graft preservation: a role for melatonin and trimetazidine cocktail. J Pineal Res 55: 65-78. [Crossref]

31. Ruixing Y, Wenwu L, Al-Ghazali R (2007) Trimetazidine inhibits cardiomyocyte apoptosis in a rabbit model of ischemiareperfusion. Transl Res 149: 152-160. [Crossref]

32. Dehina L, Vaillant F, Tabib (2013) Trimetazidine demonstrated cardioprotective effects through mitochondrial pathway ina model of acute coronary ischemia. Naunyn Schmiedebergs Arch Pharmacol 386: 205-215. [Crossref]

33. Zaouali MA, Ben Mosbah I, Boncompagni E (2010) Hypoxia inducible factor-1alpha accumulation in steatotic liver preservation: role of nitric oxide. World J Gastroenterol 16: 3499-3509. [Crossref]

34. Settaf A, Zaim N, Bellouch M, Tillement JP, Morin D (2001) Trimetazidine prevents ischemia-reperfusion injury in hepatic surgery under vascular clamping. Therapie 56: 569-574. [Crossref]

35. Baker TA, Geng Q, Romero J, Picken MM, Gamelli RL, et al. (2010) Prolongation of myocardial viability by proteasome inhibition during hypothermic organ preservation. Biochem Biophys Res Comm 401: 548-553. [Crossref]

36. Bejaoui M, Zaouali MA, Folch-Puy E (2014) Bortezomib enhances fatty liver preservation in Institut George Lopez-1 solution through adenosine monophosphate activated protein kinase and Akt/mTOR pathways. Journal Pharm Pharmacol 66: 6272. [Crossref]

37. Lemaire F, Sigrist S, Delpy E (2019) Beneficial effects of the novel marine oxygen carrier M101 during cold preservation of rat and human pancreas. J Cell Mol Med 23 . 8025-8034. [Crossref]

38. Le Gall T, Polard V, Rousselot M (2014) In vivo biodistribution and oxygenation potential of a new generation of oxygen carrier. J Biotechnol 187: 1-9. [Crossref]

39. Mallet V, Dutheil D, Polard V (2014) Dose-ranging study of the performance of the natural oxygen transporter HEMO2 Life in organ preservation. Artif Organs 38: 691-701. [Crossref]

40. Thuillier R, Dutheil D, Trieu MT (2011) Supplementation with a new therapeutic oxygen carrier reduces chronic fibrosis and organ dysfunction in kidney static preservation. Am J Transplant 11: 1845-1860. [Crossref]

41. Glorion M, Polard V, Favereau F (2018) Prevention of ischemiareperfusion lung injury during static cold preservation by supplementation of standard preservation solution with HEMO2life((R)) in pig lung transplantation model. Artif Cells Nanomed Biotechnol 46: 1773-1780. [Crossref]

42. Kaminski J, Hannaert P, Kasil A (2019) Efficacy of the natural oxygen transporter $\mathrm{HEMO} 2$ life $((\mathrm{R}))$ in cold preservation in a preclinical porcine model of donation after cardiac death. Transpl Int 32: 985-996. [Crossref]

43. Kasil A, Giraud S, Couturier P (2019) Individual and combined impact of oxygen and oxygen transporter supplementation during kidney machine preservation in a porcine preclinical kidney transplantation model. Int J Mol Sci 20: 1992 [Crossref]

44. Ben Gdara N (2013) Etude de l'effet protecteur du monoxyde d'azote sur la reprise fonctionnelle du foie en ischémie froide. Master de recherche. Faculté de Pharmacie de Monastir (Tunisie).

Copyright: (C2020 Gdara NB. This is an open-access article distributed under the terms of the Creative Commons Attribution License, which permits unrestricted use, distribution, and reproduction in any medium, provided the original author and source are credited. 